\title{
Stochastic unraveling of Redfield master equations and its application to electron transfer problems
}

\author{
Ivan Kondov, ${ }^{1, *}$ Ulrich Kleinekathöfer, ${ }^{2}$ and Michael Schreiber ${ }^{1}$ \\ ${ }^{1}$ Institut für Physik, Technische Universität, 09107 Chemnitz, Germany \\ ${ }^{2}$ International University Bremen, P.O.Box 750 561, 28725 Bremen, Germany
}

\begin{abstract}
A method for stochastic unraveling of general time-local quantum master equations (QMEs) is proposed. The present kind of jump algorithm allows a numerically efficient treatment of QMEs which are not in Lindblad form, i.e. are not positive semidefinite by definition. The unraveling can be achieved by allowing for trajectories with negative weights. Such a property is necessary, e.g. to unravel the Redfield QME and to treat various related problems with high numerical efficiency. The method is successfully tested on the damped harmonic oscillator and on electron transfer models including one and two reaction coordinates. The obtained results are compared to those from a direct propagation of the reduced density matrix (RDM) as well as from the standard quantum jump method. Comparison of the numerical efficiency is performed considering both the population dynamics and the RDM in the Wigner phase space representation.
\end{abstract}

PACS numbers: 03.65.Yz, 42.50.Lc, 34.70.+e, 82.20.-w 


\section{INTRODUCTION}

Time-independent as well as time-dependent phenomena in chemical physics, quantum optics, solid state physics, biological physics, etc. are often described using QMEs 1.2 . In particular, electron transfer (ET) dynamics in open quantum systems, i.e., systems with dissipation, can be conveniently treated within this formalism. The QMEs govern the time evolution of density matrices which are used in order to represent the mixed nature of the states. Recently stochastic wave function methods have received a great deal of attention. In unraveling schemes one considers an ensemble of stochastic Schrödinger equations (SSEs) which in the limit of a large ensemble resemble the respective QME. Although all SSE approaches have a common basis ${ }^{3}$ they are usually divided into two classes. One is the quantum jump method also known as Monte Carlo wave function (MCWF) approach 4,5.6.7.8. In this approach the dynamics is described by a Schrödinger-like wave equation interrupted by instantaneous deviations from the continuous motion (quantum jumps). The second class of SSE approaches are the quantum diffusion models with continuous motion ${ }^{9.10}$ which are not in the center of interest here. The numerical effort for solving SSEs scales much more favorably with the size of the basis than a direct propagation of a density matrix since one is dealing with wave functions and not density matrices (for a comparison of direct integrators, see Ref. 11). Thus, stochastic unraveling is an efficient numerical tool for solving QMEs. Of course, to achieve good statistics one has to average over a large number of wave functions. So the SSE approaches become preferable for large and complex systems with many degrees of freedom. In passing, we want to mention that in the present paper we are not interested in establishing a relation between the SSE dynamics and some measurement process. Thus, the treatment of single trajectories will be done without giving a special physical meaning to them.

One of the important properties of a density matrix is the positive semi-definiteness for all times, i.e. that all populations are positive or zero. This property is fulfilled for QMEs of the Lindblad form 12 but not necessarily for reduced dynamics in general ${ }^{13,14}$. A slightly generalized generator for a completely positive density-matrix evolution can be found in Ref. 15 while a discussion on the non-Markovian case has been done in Ref. 16. Most of the unraveling schemes ${ }^{4,6.9,10,17.18}$ have been restricted to QMEs of Lindblad form ${ }^{12}$ which ensures that the RDM stays positive semidefinite for all times and all parameters. Nevertheless, there are many physically meaningful QMEs which result in positive semidefinite RDMs

although they are not of Lindblad form 19.20 . The increasing interest in descriptions beyond the Lindblad class such as the quantum Brownian motion ${ }^{19,21}$, the Redfield formalism ${ }^{2,22}$, non-Markovian schemes 23.24 .25 , etc. resulted in various efforts to develop new stochastic wave function algorithms.

Strunz et al: $\stackrel{19.21}{ }$ extended the QME for Brownian motion to a non-Markovian QME and then applied a quantum state diffusion algorithm. A similar approach was also proposed by Gaspard et al. ${ }^{26}$. Recently Stockburger and Grabert ${ }^{27}$ developed a method for an exact formulation of the RDM in terms of SSEs of a system coupled to a linear heat bath. Breuer et $a l{ }^{25}$ extended a scheme which they had used to calculate multi-time correlation functions ${ }^{28}$ to the unraveling of QMEs. Their technique is based on doubling the Hilbert space. So 
instead of a single stochastic wave function one has a pair of them ${ }^{25}$. In this approach, norm and Hermiticity are not preserved in single realizations but only in the ensemble average which makes the algorithm unstable. Since stability and efficiency are crucial issues for unraveling algorithms we propose an alternative approach which fulfills these criteria. Though the present approach has only been tested for Redfield and Brownian-dynamics master equations so far 29 there are only few restrictions to its range of validity and it is therefore applicable to a much larger class of time-local quantum master equations.

ET is commonly treated in modern theories with use of the RDM formalism and QMEs. Alternatives to QMEs are, for example, semi-classical theories ${ }^{30}$, path integral methods ${ }^{31.32}$ and recently the self-consistent hybrid approach33.34.35. The latter was shown to treat successfully the spin-boson problem ${ }^{33}$, the ET in mixed-valence compounds ${ }^{34.35}$ as well as the heterogeneous ET at semiconductor surfaces ${ }^{35}$. Solving the QME for ET in model systems with one $36,37,38,39$ and many reaction coordinates 40 has been done with success. Exhaustive reviews on ET can be found, e.g., in Refs. 41 and 42. Apart from the non-Markovian descriptions of transfer phenomena 23.43 .44 .45 the use of Redfield theory for ET was investigated as well11,37,38,46,47,48,49. The model used in the latter references is based on vibronically coupled diabatic potentials which are sufficiently well approximated by harmonic potentials. In particular, the influence of the electronic coupling between the diabatic states on the dissipation was investigated. Neglecting this effect results in the diabatic damping approximation (DDA) $\stackrel{46.47}{\longrightarrow}$. This approximation as well as considering first order perturbation theory in the electronic coupling were objects of recent studies ${ }^{47}$. A typical problem that occurs with increasing the complexity of the ET models, i.e. the dimension of the RDM, is the numerical effort. Thus, the stochastic unraveling of generalized time-local QME was developed with the prospect of applications to more complex ET systems.

Recently the present scheme was briefly demonstrated for the quantum Brownian motion of a harmonic oscillator ${ }^{29}$. In the present paper the stochastic unraveling of the Redfield QME shall be considered in more detail as well as applications concerning multi-mode models for ET shall be presented. In the next Section a brief introduction to the Redfield formalism will be given. Section III focuses on the derivation of the SSEs relevant for the generalized time-local QME while Section [V] will provide explicit expressions for the jump rates. In Section $\nabla$ we describe three concrete applications of the proposed quantum jump method: the damped harmonic oscillator and a model for ET with one and two reaction modes. A study and discussion of the numerical efficiency in Section VI and a conclusion follow.

The detailed quantum jump algorithm used in the present contribution can be found in the Appendix. Atomic units are used throughout the paper, i.e. $\hbar=1$.

\section{REDFIELD FORMALISM}

In Redfield theory the overall system is partitioned into a relevant system whose evolution is of interest and a thermal bath using the Hamiltonian

$$
H=H_{\mathrm{S}}+H_{\mathrm{B}}+H_{\mathrm{SB}} .
$$


Here $H_{\mathrm{S}}$ and $H_{\mathrm{B}}$ are the Hamiltonians of the relevant system and of the bath while $H_{\mathrm{SB}}$ describes their interaction. In Subsec. $\nabla \mathrm{V}$ it will be shown how this partitioning can be rigorously performed. In general, the interaction part can be represented by bilinear products

$$
H_{\mathrm{SB}}=\sum_{m} \Phi_{m} K_{m}
$$

of system and bath operators, $K_{m}$ and $\Phi_{m}$, respectively. In the following $K_{m}$ and $\Phi_{m}$ will be considered Hermitian. The state of the system is described by the RDM performing a trace of the total density matrix $\sigma$ over the bath degrees of freedom, i.e. $\rho=\operatorname{tr}_{\mathrm{B}} \sigma$. It is assumed that the bath stays in thermodynamic equilibrium at all times. This means that the relaxation of the bath is much faster than the evolution of the system. In addition, one assumes that the system-bath interaction is sufficiently small to be treated perturbatively to second order. Using the Hamiltonian (II) and the assumptions described above one obtains a non-Markovian QME for the RDM. One possible way to obtain a Markovian QME instead is to neglect memory effects which are due to the finite bath correlation time. The formal treatment yields the Redfield QME ${ }^{2.50}$

$$
\dot{\rho}=-i\left[H_{\mathrm{S}}, \rho\right]+\sum_{m}\left\{\left[\Lambda_{m} \rho, K_{m}\right]+\left[K_{m}, \rho \Lambda_{m}^{\dagger}\right]\right\} .
$$

Note that the operator $\Lambda_{m}^{\dagger}$ is the adjoint of the relaxation operator $\Lambda_{m}$. This is only true if $K_{m}$ and $\Phi_{m}$ are Hermitian ${ }^{2.14}$ but not in general ${ }^{22.50}$. The relaxation operator is given by

$$
\Lambda_{m}=\sum_{n} \int_{0}^{\infty} d \tau C_{m n}(\tau) K_{n}^{\mathrm{I}}(-\tau),
$$

where $C_{m n}(\tau)$ is the bath correlation function and $K_{n}^{\mathrm{I}}$ the system operator in the interaction picture. Usually it is easier to obtain the latter quantity in the frequency domain, e.g. with use of molecular dynamics simulations or with a simple bath modeling. Either approach yields the bath spectral density $J_{m n}(\omega)$ in terms of which the correlation function can be constructed as ${ }^{2}$

$$
C_{m n}(\omega)=2 \pi[1+n(\omega)]\left[J_{m n}(\omega)-J_{m n}(-\omega)\right]
$$

with the Bose-Einstein distribution $n(\omega)=\left(e^{\beta \omega}-1\right)^{-1}$ and $\beta=\left(k_{\mathrm{B}} T\right)^{-1}$ being the inverse temperature. All considerations in the present work will be limited to the Ohmic form of the spectral density with exponential cut-off. However, a spectral density of Debye form can be constructed which results in nearly the same values of $J_{m n}(\omega)$ for the specific spectrum of $H_{\mathrm{S}}$ used.

Under certain approximations Eq. (3) can be transformed to Lindblad form ${ }^{46}$

$$
\frac{d \rho(t)}{d t}=-i\left[H_{\mathrm{S}}, \rho(t)\right]+\sum_{n}\left[L_{n} \rho(t) L_{n}^{\dagger}-\frac{1}{2} \rho(t) L_{n}^{\dagger} L_{n}-\frac{1}{2} L_{n}^{\dagger} L_{n} \rho(t)\right] .
$$

One way to obtain the Lindblad QME (6) is starting either from the non-Markovian QME or from Eq. (44) and assuming a $\delta$-correlated bath ${ }^{2.14}$, i.e., $C_{m n}(\tau) \rightarrow c_{m n} \delta(\tau)$. Subsequent 
diagonalization of the correlation matrix $\boldsymbol{c}$ by means of a unitary transformation $\boldsymbol{c}=U^{\dagger} \boldsymbol{\kappa} U$, yields Eq. (6) with $L_{n}=\sqrt{\kappa_{n}} \sum_{m} U_{n m} K_{m}$. Alternatively, the Lindblad QME is obtained when the DDA ${ }^{46.47}$ is invoked with the so-called rotating wave approximation (RWA) in the system-bath coupling as done in the present paper. The explicit form of the corresponding Lindblad operators used will be given in the sections below.

The Lindblad QME (6) will be used to compare the numerical efficiency of the present quantum jump method method with the standard one 4.6 .9 .10 .17 by solving the single-mode and the two-mode ET model. For more details of the DDA we refer to Refs. 46 and 47.

\section{STOCHASTIC SCHRÖDINGER EQUATION}

To start with the derivation of the unraveling scheme we first like to formulate the timelocal QME in its most general form

$$
\begin{aligned}
\frac{d \rho(t)}{d t}= & A(t) \rho(t)+\rho(t) A^{\dagger}(t) \\
& +\sum_{k=1}^{M}\left\{C_{k}(t) \rho(t) E_{k}^{\dagger}(t)+E_{k}(t) \rho(t) C_{k}^{\dagger}(t)\right\} .
\end{aligned}
$$

Because $A(t), C_{k}(t)$, and $E_{k}(t)$ are arbitrary operators this equation conserves only Hermiticity. In order to conserve also the norm further restrictions have to be applied as shown below. All time arguments will be dropped henceforth for clarity.

The RDM will be recovered by averaging over an ensemble of two vectors $|\psi\rangle$ and $|\phi\rangle$, which are elements of the doubled Hilbert space, as

$$
\rho=\overline{|\psi\rangle\langle\phi|}+\overline{|\phi\rangle\langle\psi|} \text {. }
$$

Every individual realization of the stochastic process before averaging denoted by the pair $(|\psi\rangle,|\phi\rangle)$ will be called a trajectory. In contrast to Ref. 25 the averaging formula (8) preserves Hermiticity of single trajectories leading to a significantly improved numerical performance of the scheme. Each trajectory $(|\psi\rangle,|\phi\rangle)$ is propagated be means of two SSEs having the following generic form

$$
\begin{aligned}
& d|\psi\rangle=D_{1}|\psi\rangle d t+\sum_{k=1}^{M} \sum_{i=1}^{2} S_{1 k}^{i}|\psi\rangle d \xi_{k}^{i}, \\
& d|\phi\rangle=D_{2}|\phi\rangle d t+\sum_{k=1}^{M} \sum_{i=1}^{2} S_{2 k}^{i}|\phi\rangle d \xi_{k}^{i}
\end{aligned}
$$

Unlike deterministic differential equations the SSEs include differentials of the complex noise variables $\xi_{k}^{i}$ in addition to the time variable. The superscript in $\xi_{k}^{i}$ denotes which of the two terms from the Hermitian pair in the sum in Eq. (7) is taken while the subscript counts the relevant dissipative channels from 1 to $M$. The operators $D_{1}$ and $D_{2}$ specify the deterministic and the operators $S_{j k}^{i}$ the stochastic part of the evolution. In general, they 
may be time-dependent. The stochastic differentials ${ }^{51} d \xi_{k}^{i}$ are assumed to have zero mean, to be uncorrelated and normalized to $d t$ :

$$
\overline{d \xi_{k}^{i}}=0, \overline{d \xi_{k}^{i *} d \xi_{l}^{j}}=\delta_{i j} \delta_{k l} d t
$$

Differentiating Eq. (8), neglecting all terms higher than first order in $d t$, and assuming that ensemble averages always factorize $\mathrm{e}^{\underline{3}}$ yields

$$
\begin{aligned}
d \rho= & {\left[D_{1} \overline{|\psi\rangle\langle\phi|}+D_{2} \overline{|\phi\rangle\langle\psi|}\right] d t } \\
& +\sum_{k=1}^{M}\left[S_{1 k}^{1}\left|\overline{\psi\rangle\langle\phi|} S_{2 k}^{1 \dagger}+S_{2 k}^{2}\right| \overline{\phi\rangle\langle\psi|} S_{1 k}^{2 \dagger}\right] d t+\text { H.c. }
\end{aligned}
$$

Comparing Eq. (11) with the original QME (7) one is able to replace

$$
S_{1 k}^{2}=S_{2 k}^{1}=C_{k}+\alpha_{k}^{1} \quad \text { and } \quad S_{1 k}^{1}=S_{2 k}^{2}=E_{k}+\alpha_{k}^{2}
$$

where $\alpha_{k}^{1}$ and $\alpha_{k}^{2}$ are arbitrary possibly time-dependent scalar functions of $(|\psi\rangle,|\phi\rangle)$. Plugging the latter expressions into Eq. (11) yields

$$
D_{1}=D_{2}=A-\sum_{k=1}^{M}\left(\alpha_{k}^{2 *} C_{k}+\alpha_{k}^{1 *} E_{k}+\alpha_{k}^{1} \alpha_{k}^{2 *}\right) .
$$

According to Ref. 3 Eq. (9) describes a quantum diffusion process if the leading terms in $d \xi_{k}^{i}$ are of first order in $\sqrt{d t}$. When $d \xi_{k}^{i}$ can be given by a finite number of values only, e.g. $\pm \sqrt{d t}$, the process results in continuous but random trajectories within each infinitesimal time interval $d t$ (for $d t \rightarrow 0$ the trajectories become smooth but still stay noisy). In that way one derives diffusion methods which will not be considered in the present work. However, if the leading terms in $d \xi_{k}^{i}$ have finite values of order unity, i.e. zeroth order in $\sqrt{d t}$, Eq. (9) leads to the so-called quantum jump methods which produce trajectories that are deterministic during finite time intervals connected by discontinuous transitions (jumps). The jumps are specified by their jump rates $p_{k}^{i}$, which have to be real scalar functions of $(|\psi\rangle,|\phi\rangle)$. If $n_{k}^{i}(t)$ is the number of jumps in channel $k$ due to term $i$ up to time $t$, the probability for $n_{k}^{i}(t)$ to increase by one, i.e. the expectation value of both $d n_{k}^{i}$ and $\left(d n_{k}^{i}\right)^{2}$, should be equal to $p_{k}^{i} d t$ during the infinitesimal time interval $d t$. This can be written $a_{s}{ }^{3}$

$$
d \xi_{k}^{i}=\frac{d n_{k}^{i}-p_{k}^{i} d t}{\sqrt{p_{k}^{i}}} e^{i \varphi}
$$

so that it obeys condition (10). The phase factor $e^{i \varphi}$ leads merely to a phase shift in the wave vectors and cancels within each realization and we therefore set $\varphi=0$. If $d n_{k}^{i}$ vanishes for all $k$ and $i$, then Eq. (9) becomes a deterministic Schrödinger equation. For any $k$ and $i, d n_{k}^{i}=1$ indicates the occurrence of a jump. In this case we have $d(|\phi\rangle,|\psi\rangle)=$ $(|\phi\rangle,|\psi\rangle)_{\text {after jump }}-(|\phi\rangle,|\psi\rangle)_{\text {before jump. Taking this into account and substituting Eqs. (14) }}$ and (12) into Eq. (9) it is found that $\alpha_{k}^{i}=-\sqrt{p_{k}^{i}}$. Eventually, the final form of the SSEs 
for the quantum jump method is obtained as

$$
\begin{aligned}
d|\psi\rangle= & \left(A+\sum_{k=1}^{M} \frac{p_{k}^{1}+p_{k}^{2}}{2}\right)|\psi\rangle d t \\
& +\sum_{k=1}^{M}\left[\left(\frac{E_{k}}{\sqrt{p_{k}^{1}}}-1\right) d n_{k}^{1}+\left(\frac{C_{k}}{\sqrt{p_{k}^{2}}}-1\right) d n_{k}^{2}\right]|\psi\rangle, \\
d|\phi\rangle= & \left(A+\sum_{k=1}^{M} \frac{p_{k}^{1}+p_{k}^{2}}{2}\right)|\phi\rangle d t \\
& +\sum_{k=1}^{M}\left[\left(\frac{C_{k}}{\sqrt{p_{k}^{1}}}-1\right) d n_{k}^{1}+\left(\frac{E_{k}}{\sqrt{p_{k}^{2}}}-1\right) d n_{k}^{2}\right]|\phi\rangle .
\end{aligned}
$$

\section{JUMP RATES}

Essential for the performance and particularly for the convergence behavior of the quantum jump method is how the jump rates $p_{k}^{1}$ and $p_{k}^{2}$ are specified. They have no physical meaning since in the average they do not influence any observable but determine the statistical error. A detailed discussion on the optimization of the jump rates as free parameters can be found in Ref. 52. Another freedom is that Eq. (77) is invariant with respect to a gauge transformation of the kind $C_{k} \rightarrow f C_{k}, E_{k} \rightarrow E_{k} / f$ if $f$ is a real, scalar function of time. Each single realization, and hence the stochastic process, is independent of this gauge transformation and using such transformation offers us no further advantages. We note that the jump rates in Ref. 25 do not fulfill the invariance under this gauge transformation.

Following the approach in Ref. 52 we require that the norm of every single trajectory is constant in time. Under such a condition expressed as

$$
\operatorname{tr}\left\{\frac{d}{d t}[|\psi\rangle\langle\phi|+| \phi\rangle\langle\psi|]\right\}=0
$$

the $p_{k}^{i}$ are adapted at each moment of time. This approach yields a numerically stable and efficient algorithm. In contrast, numerical tests with jump rates adapted to other quantities

such as $\langle\phi \mid \phi\rangle,\langle\psi \mid \psi\rangle$, etc. resulted in an unstable scheme. The operators that enter the QME (17) are restricted by condition (16) yielding

$$
A+A^{\dagger}+\sum_{k=1}^{M}\left(E_{k}^{\dagger} C_{k}+C_{k}^{\dagger} E_{k}\right)=0
$$

Let us try to determine the jump rates from this condition. The total jump rate is obtained applying Eq. (16) to the deterministic part of Eq. (15):

$$
\tilde{p}=-\frac{\left\langle\phi\left|A+A^{\dagger}\right| \psi\right\rangle+\left\langle\psi\left|A+A^{\dagger}\right| \phi\right\rangle}{\langle\phi \mid \psi\rangle+\langle\psi \mid \phi\rangle} .
$$


All partial jump rates can be then successively found using Eqs. (17) and (18):

$$
\begin{aligned}
& \tilde{p}_{k}^{1}=\frac{\left\langle\phi\left|C_{k}^{\dagger} E_{k}\right| \psi\right\rangle+\left\langle\psi\left|E_{k}^{\dagger} C_{k}\right| \phi\right\rangle}{\langle\phi \mid \psi\rangle+\langle\psi \mid \phi\rangle}, \\
& \tilde{p}_{k}^{2}=\frac{\left\langle\phi\left|E_{k}^{\dagger} C_{k}\right| \psi\right\rangle+\left\langle\psi\left|C_{k}^{\dagger} E_{k}\right| \phi\right\rangle}{\langle\phi \mid \psi\rangle+\langle\psi \mid \phi\rangle} .
\end{aligned}
$$

Here a problem occurs because the values of the $\tilde{p}_{k}^{i}$ do not have to be positive for all trajectories at all times. But, since the jump rates $p_{k}^{i}$ are arbitrary real functions we can choose them as the absolute values of the $\tilde{p}_{k}^{i}$

$$
\begin{aligned}
& p_{k}^{1}=\left|\frac{\left\langle\phi\left|C_{k}^{\dagger} E_{k}\right| \psi\right\rangle+\left\langle\psi\left|E_{k}^{\dagger} C_{k}\right| \phi\right\rangle}{\langle\phi \mid \psi\rangle+\langle\psi \mid \phi\rangle}\right|, \\
& p_{k}^{2}=\left|\frac{\left\langle\phi\left|E_{k}^{\dagger} C_{k}\right| \psi\right\rangle+\left\langle\psi\left|C_{k}^{\dagger} E_{k}\right| \phi\right\rangle}{\langle\phi \mid \psi\rangle+\langle\psi \mid \phi\rangle}\right| .
\end{aligned}
$$

An additional weight factor \pm 1 for the trajectories has to be introduced which changes its sign every time a jump is performed with $p_{k}^{i}=-\tilde{p}_{k}^{i}$. It can also be implemented (as in the appendix) by allowing for negative norms of the trajectories. The change from $\tilde{p}_{k}^{i}$ to $p_{k}^{i}$ gives rise to a small deviation of the norm from unity because in the regions where not all $\tilde{p}_{k}^{i}$ and $p_{k}^{i}$ are identical, norm conservation is no longer guaranteed, i.e. the sum of the $p_{k}^{i}$ differs from $\tilde{p}$. As long as the occurrence of a jump is a very rare event and the number of negative $\tilde{p}_{k}^{i}$ is also very small the deviation from the initial norm is expected to be small. In all tests this deviation was far below $1 \%$ without effecting the numerical efficiency of the proposed algorithm. This is how the scheme tolerates trajectories with possibly negative weights which arise from the fact that the RDM with the QME (7) is not necessarily positive semidefinite. If the RDM stays positive semidefinite during its entire time evolution the negative weights are not needed, i.e. all trajectories can be normalized to unity and represent physically relevant pure states. A possible implementation of the present unraveling scheme is shown in the appendix.

In the examples below the RDM can exhibit negative eigenvalues. This nonphysical situation could probably be improved by applying an initial slippage to the initial state ${ }^{14}$. Another possibility to avoid non-positive semidefinite RDMs is to start with a derivation of different QMEs in the form (77) with time-dependent coefficients. It has been shown that non-Lindblad QMEs with time-dependent coefficients can preserve the positivity of the $\mathrm{RDM}^{19.20}$. Nevertheless, an unraveling scheme has to be able to follow also the nonphysical behavior of the QME because in the ensemble average the solution of the SSEs should completely coincide with the exact solution of the QME. 


\section{APPLICATIONS OF THE PRESENT UNRAVELING METHOD}

\section{A. Damped harmonic oscillator}

One of the most simple toy models used for testing in dissipative quantum dynamics is the damped harmonic oscillator. Here it will be formulated within Redfield theory, i.e. one has to obtain the explicit form of the operators in Eq. (3). The oscillator has mass $\mathcal{M}$ and frequency $\omega_{0}$. If the thermal bath is modeled by quantum harmonic oscillators and the system operator $K$ is the oscillator coordinate $q=\left(a+a^{\dagger}\right) / \sqrt{2 \mathcal{M} \omega_{0}}$ the sum in Eq. (3) contains only one term in which

$$
\Lambda=\Gamma \sqrt{\frac{\mathcal{M} \omega_{0}}{2}}\left[\left(n\left(\omega_{0}\right)+1\right) a+n\left(\omega_{0}\right) a^{\dagger}\right] .
$$

The damping rate $\Gamma$ is related to the spectral density of the bath $J(\omega)$ as $\Gamma=\pi J\left(\omega_{0}\right) /\left(\mathcal{M} \omega_{0}\right)$. Therefore, the explicit form of $J(\omega)$ is not necessary since the oscillator and the bath interchange quanta only at the frequency $\omega_{0}$. Performing either the RWA ${ }^{53,54}$ or the secular approximation 2.54 .55 Eq. (3) is transformed into a Lindblad QME (6). For the sake of simplicity this will be shown here with the RWA for the harmonic oscillator but the generalization for the ET model solved within the DDA is straightforward. Inserting the expressions for $\Lambda$ and $K$ into Eq. (3), denoting $b_{1}=a$ and $b_{2}=a^{\dagger}$, and performing some calculus the QME obtains the form

$$
\dot{\rho}=-i\left[H_{\mathrm{S}}, \rho\right]+\frac{\Gamma}{2} \sum_{i, j=1}^{2} \kappa_{i j}\left(b_{i} \rho b_{j}^{\dagger}-\frac{1}{2} b_{j}^{\dagger} b_{i} \rho-\frac{1}{2} \rho b_{j}^{\dagger} b_{i}\right),
$$

where $\boldsymbol{\kappa}$ is the correlation matrix

$$
\boldsymbol{\kappa}=\left(\begin{array}{cc}
n\left(\omega_{0}\right) & n\left(\omega_{0}\right)+\frac{1}{2} \\
n\left(\omega_{0}\right)+\frac{1}{2} & n\left(\omega_{0}\right)+1
\end{array}\right)
$$

In order to transform Eq. (22) into Lindblad form either $\boldsymbol{\kappa}$ has to be diagonalized imposing conditions for which the eigenvalues are positive ${ }^{56}$ or the RWA in the system-bath coupling ${ }^{54}$ has to be performed. It is easily seen that the determinant of $\boldsymbol{\kappa}$ is $-\frac{1}{4}$ and hence the former method fails for this QME. Performing the RWA implies that the off-diagonal elements of $\boldsymbol{\kappa}$ are set to zero. Then the Lindblad operators take the explicit form

$$
L_{1}=\sqrt{\left(n\left(\omega_{0}\right)+1\right) \Gamma} a \text { and } L_{2}=\sqrt{n\left(\omega_{0}\right) \Gamma} a^{\dagger} .
$$

This result is easily generalized for the ET models discussed in the next two subsections. $L_{1}$ and $L_{2}$ have a clear physical interpretation. $L_{1}$ damps all occupied levels bringing their populations one level lower, while $L_{2}$ has the opposite effect. In the thermodynamic equilibrium the jump rates for both operators are equal: the populations do not change.

To find the operators involved in the generalized QME (17) (with $M=1$ for the harmonic oscillator) one has to carry out the commutators in Eq. (3). Then one can easily identify

$$
C_{1}=K, E_{1}=\Lambda, A=-i H_{\mathrm{S}}-K \Lambda \text {. }
$$


In contrast to the Lindblad operators (24) the action of $C_{1}$ and $E_{1}$ on the wave function is more subtle. This is why it is difficult, and probably not possible, to assign a certain physical process to a single trajectory.

In our stochastic simulation of the damped harmonic oscillator the temperature $T=\omega_{0} / 4$ and $\Gamma=\omega_{0} / 10$ are used. Figure 1] shows the population dynamics of the lowest four levels of the oscillator starting from the pure initial state $\rho_{33}=1$. As seen, the convergence to the exact solution is very slow $\left(10^{4}\right.$ trajectories are still not sufficient). On the other hand the test system is very small and the QME can be solved very fast using direct propagators. The true advantage of the method can be seen with larger systems, where it shows both a faster convergence and a good scaling.

\section{B. Electron transfer model with one reaction coordinate}

Let us consider a model for electron transfer with the Hamiltonian $\underline{47}$

$$
H_{\mathrm{S}}=H^{(0)}+V=\sum_{i} H_{i}|i\rangle\langle i|+V
$$

where $H_{i}$ are the Hamiltonians of two harmonic oscillators (i.e., $i=1,2$ ) which describe the vibronic spectrum of two electronic states interacting via the electronic coupling $V$. If the system includes a single reaction coordinate $q$ the vibronic Hamiltonians read

$$
H_{i}=U_{i}+\omega_{i}\left(a_{i}^{\dagger} a_{i}+\frac{1}{2}\right)+\frac{\omega_{i} \Delta_{i}}{\sqrt{2}}\left(a_{i}+a_{i}^{\dagger}\right)
$$

where $a_{i}$ and $a_{i}^{\dagger}$ are the boson operators, $\Delta_{i}$ the dimensionless displacements of the harmonic potentials along the reaction coordinate, $\omega_{i}$ the oscillator frequencies, and $U_{i}$ the electronic excitation energies. A very useful parameter of the system which is related to the last term in Eq. (27) is the reorganization energy $\lambda_{i}=\omega_{i} \Delta_{i}^{2} / 2$. It is also proportional to the vibronic coupling $\omega_{i} \Delta_{i}$. Using the former expression one can define the potential minima as $U_{i}^{0}=U_{i}-\lambda_{i}$. Configurations in which the potential minimum of the upper free-energy surface is lower in energy than the lower free-energy surface at that point are in the socalled normal region. If the opposite is true the configuration is in the Marcus inverted region. The electronic coupling $v_{12}$ between the model potential surfaces is independent of the coordinate. So the respective term in Eq. (26) obtains the explicit form

$$
V=\sum_{i, j} \sum_{M, N}\left(1-\delta_{i j}\right) v_{i j} f(i, M ; j, N)|i M\rangle\langle j N| \text {. }
$$

The Franck-Condon factors $f(i, M ; j, N)$ are calculated using the eigenfunctions $\varphi_{i M}$ of the harmonic oscillators

$$
f(i, M ; j, N)=\langle i M \mid j N\rangle=\int d q \varphi_{i M}(q) \varphi_{j N}(q) .
$$

By analogy with the damped harmonic oscillator the system operator $K$ is defined as the coordinate operator, i.e.

$$
K=q=\sum_{i}\left(2 \omega_{i} \mathcal{M}\right)^{-1 / 2}\left(a_{i}^{\dagger}+a_{i}\right)|i\rangle\langle i| .
$$


We consider a potential configuration in the normal region with no barrier between the two harmonic potentials which have equal curvature $\left(\omega_{1}=\omega_{2} \equiv \omega_{0}\right)$, change of free energy $U_{2}^{(0)}-U_{1}^{(0)}=-2 \omega_{0}$, reorganization energy $\lambda_{1}=3 \omega_{0}, \lambda_{2}=0$ and inter-center coupling $v_{12}=\omega_{0}$. The reason for this choice is the intention to compare the standard and the new quantum jump methods. As the Lindblad QME is obtainable only with RWA and DDA one has to study a parameter region where both QMEs generate almost the same dynamics. The bath is described by an Ohmic spectral density with cut-off frequency $\omega_{c}=\omega_{0}$ at temperature $k_{\mathrm{B}} T=\omega_{0} / 4$. The system-bath interaction is characterized by the damping rate $\Gamma=\pi \eta /(\mathcal{M} \exp (1))=\omega_{0} / 10$ (see Ref. 47 for details). Again, the operators necessary for the new quantum jump method are defined according to Eq. (25).

A Gaussian wave packet located at the donor state $|1\rangle$ having energy slightly above the crossing of the harmonic potentials was chosen as initial state. The numerical simulation with about 1000 trajectories provides sufficiently converged and accurate results. Figure 2 shows the relaxation of the ensemble-averaged donor population $\overline{P_{1}}=\overline{\langle\psi \mid 1\rangle\langle 1 \mid \phi\rangle}+\overline{\langle\phi \mid 1\rangle\langle 1 \mid \psi\rangle}$. A widely discussed property of the Redfield equation is that it does not strictly conserve positivity of the $\mathrm{RDM}{ }^{54}$. Although $\overline{P_{1}}$ is always positive the tiny negative fraction in Fig. 3 is an evidence for the existence of single realizations with negative $P_{1}$. In contrast, the simulation of the same system with the Lindblad operators (24) by means of the standard quantum jump method ${ }^{4.69 .10 .17}$ keeps all values of $P_{1}$ well confined between 0 and 1 .

Besides numerical efficiency, another advantage of the quantum trajectories is the better insight into the quantum mechanisms underlying the overall dynamics of the ensemble. Though it is impossible to give a direct physical interpretation of every single trajectory one can extract information from the ensemble statistics. As we can see in Fig. [3 the distribution of the individual expectation values of the population is skew and comprises several maxima. One can better visualize the wave packet dynamics in phase space using the Wigner representation of the $\mathrm{RDM}^{57}$ as done in Figs. 4 and 5 . The evolution of the expectation values of the momentum and the coordinate, which can be regarded as the center of mass of the wave packet, is described by a path in phase space as shown in Fig. 6.

The wave packet starts off with zero momentum from the location of the excited state. One can distinguish two stages of the ET dynamics. In the first stage the wave packet splits into several parts and occupies the whole accessible phase volume, i.e., it spreads (see Figs. [4 b, c and 國 b, c). The motion of the principal part of the wave packet, which is seen as a sharp peak in Fig. [3, implies coherent transfer of population. The peak moves rapidly with time in an oscillatory fashion while its amplitude decays as decoherence processes advance. After that all parts of the wave packet coalesce to a single bell-shaped distribution. In the second stage the wave packet continues to propagate slowly in phase space while its maximum is approaching the equilibrium point at $\langle p\rangle_{t \rightarrow \infty}$ and $\langle q\rangle_{t \rightarrow \infty}$. This slow motion is seen as a small drift to the right beginning from the central region of the spiral path (see Fig. (6). It is due to dissipative transfer mechanisms $\underline{47}$ and is small for barrierless potential configurations as in the present case. We note also that in the time between the third population revival and the 10th vibrational period (see Fig. 2) one can recognize a crossover between the two stages discussed above. Such a moment of time is shown in Figs. 3 , 4 and 5 .

Unlike the trajectories considered in Ref. 19 within the quantum diffusion approach the 
wave packet of one individual trajectory in our calculation spreads over the whole phase volume of the system (Fig. 团a). One reason for this discrepancy is the different value used for $\hbar$. Generally, for sufficiently small $\hbar$ the trajectories become classical states and virtually shrink to points in phase space. However, the problem of their localization for small $\hbar$ is non-trivial because the system may be almost classical but with chaotic behavior.

\section{Electron transfer model with multiple reaction coordinates}

Modeling the system-bath separation one has to minimize the degrees of freedom in the relevant system and the system-bath coupling simultaneously. The small polaron transformation has been used to effectively reduce the system-bath coupling for a two-level spinboson system ${ }^{22}$. It also has been shown that this approach can be extended for multi-level systems $\underline{22}$. Alternatively, it is possible that one can successively take strongly coupled degrees of freedom from the bath and put them into the relevant-system part. This will make the effective system-bath coupling smaller and hence the application of the Redfield theory more reasonable. Multi-mode modeling of ET reactions, including systems in the inverted region, has been done in Refs. 18, 40, and 46 with similar argumentation. On the other hand, there is experimental evidence for the participation of multiple modes in the ET transition in some systems, such as oxazine- 1 in N,N-dimethylaniline $e^{\underline{58}}$ and betaine-30 in various solvents ${ }^{59}$. Correspondingly, the relevant part of the total ET system can be modeled with a treatable small set of $R$ reaction coordinates $\left\{q_{l}\right\}$. For this purpose one may select a set of representative harmonic normal modes from the molecule and from its environment (e.g. the solvent or the crystal lattice). Since all normal modes are decoupled one can use the single-mode operators to calculate the matrix elements of the necessary operators in the diabatic basis $\left|i M_{1} \ldots M_{R}\right\rangle$. The Hamiltonian of each diabatic electronic state reads

$$
H_{i}=U_{i}+\sum_{l=1}^{R}\left[\omega_{i, l}\left(a_{i, l}^{\dagger} a_{i, l}+\frac{1}{2}\right)+\frac{\omega_{i, l} \Delta_{i, l}}{\sqrt{2}}\left(a_{i, l}+a_{i, l}^{\dagger}\right)\right] .
$$

For $R$ reaction modes Eq. (2) includes $R$ summation terms linear in each coordinate $q_{l}$. The matrix element of $K_{l}$ reads

$$
\left\langle i M_{1} \ldots M_{R}\left|K_{l}\right| j N_{1} \ldots N_{R}\right\rangle=\frac{1}{\sqrt{2 \mathcal{M} \omega_{i, l}}} \delta_{i j}\left(\delta_{M_{l}+1, N_{l}} \sqrt{M_{l}+1}+\delta_{M_{l}-1, N_{l}} \sqrt{M_{l}}\right) \prod_{p \neq l} \delta_{M_{p} N_{p}}(32)
$$

where $\mathcal{M}$ is the reduced mass of the relevant system.

The multi-mode model can be easily reduced to an effective single-mode model by means of an orthogonal transformation ${ }^{60.61}$ of the Hamiltonian $H^{(0)}$. For two diabatic states with equal curvatures one can drop the electronic index. Denoting the relative displacement by $\Delta_{l}$ the transformation has the form ${ }^{61}$

$$
B_{k}=\sum_{l=1}^{R} V_{k l} a_{l}
$$


with

$$
V_{k l}=\frac{\Delta_{l} \omega_{l}}{\sqrt{2}\left(\omega_{l}-\Omega_{k}\right) D_{k}}, \quad D_{k}^{2}=\sum_{l=1}^{R} \frac{\Delta_{l} \omega_{l}}{\sqrt{2}\left(\omega_{l}-\Omega_{k}\right)^{2}}, \quad \text { for } \quad k \neq 0
$$

and

$$
V_{0 l}=\frac{\Delta_{l} \omega_{l}}{\left(\Delta_{0} \Omega_{0}\right)^{2}}, \quad\left(\Delta_{0} \Omega_{0}\right)^{2}=\sum_{l=1}^{R}\left(\Delta_{l} \omega_{l}\right)^{2}, \quad \Omega_{0}=\frac{1}{\left(\Delta_{0} \Omega_{0}\right)^{2}} \sum_{l=1}^{R} \omega_{l}^{3} \Delta_{l}^{2} .
$$

For $k \neq 0$ the new mode frequencies $\Omega_{k}$ are the roots of the equation

$$
\sum_{l=1}^{R} \frac{\Delta_{l} \omega_{l}}{\omega_{l}-\Omega_{k}}=0
$$

After this transformation the multi-mode Hamiltonian is cast into the form (11) where

$$
\begin{aligned}
H_{\mathrm{S}} & =U_{0}+\Omega_{0} B_{0}^{\dagger} B_{0}+\frac{\Omega_{0} \Delta_{0}}{\sqrt{2}}\left(B_{0}+B_{0}^{\dagger}\right) \\
H_{\mathrm{B}} & =\sum_{k=1}^{R-1} \Omega_{k} B_{k}^{\dagger} B_{k} \\
H_{\mathrm{SB}} & =\sum_{k=1}^{R-1} \frac{\Delta_{0} \Omega_{0}}{D_{k}}\left(B_{0}^{\dagger} B_{k}+B_{k}^{\dagger} B_{0}\right) .
\end{aligned}
$$

One can see that all $R$ normal modes are transformed to a finite bath with $R-1$ modes with frequencies $\Omega_{k}$. A new effective mode with frequency $\Omega_{0}$ and displacement $\Delta_{0}$ is created which is bilinearly coupled to the new bath modes. From Eq. (35) it follows that $\Delta_{0} \Omega_{0}<\sum_{l} \Delta_{l} \omega_{l}$ for positive coordinate displacements, i.e. the vibronic coupling of the new effective mode is smaller than the total vibronic coupling of the initial normal modes. Thus, a reduction from a multi-mode to a single-mode model extends the bath and enlarges the system-bath coupling. This reduction is unique. On the other hand, the addition of a bath mode to the relevant system is not unique. It depends on the choice of a certain bath mode. Nevertheless, it always reduces the system-bath coupling and enlarges the total vibronic coupling of the relevant system.

In the following, a two-mode ET model will be considered. The frequencies of the two modes were chosen 0.07 and $0.18 \mathrm{eV}$, and the reorganization energies 0.33 and $0.82 \mathrm{eV}$, respectively. These frequencies correspond to internal molecular modes in real ET systems. The effective mode was calculated using Eq. (35). The change of the free energy was taken to be $U_{2}^{0}-U_{1}^{0}=-0.2 \mathrm{eV}$ and the electronic coupling between the diabatic electronic states $v_{12}=0.1 \mathrm{eV}$. Again, a harmonic bath with an Ohmic spectral density was considered with damping rate $\Gamma=0.007 \mathrm{eV}$ and temperature $295 \mathrm{~K}$. 16 levels for each mode gave a good convergence. Initially, the lowest vibrational level of the excited state is populated (Fig. 17). The coherent dynamics of the excited state of the effective mode model yields small population in the ground state. This is followed by an almost complete revival at about 110 
fs. In contrast, the two-mode model exhibits an ultrafast coherent population transfer to the ground state. Recurrences of population in the excited state appear many times within 120 fs but their yield does not exceed $50 \%$. As the two-mode system can be regarded as a conservative two-particle system its complete recurrence period (Poincare's cycle) is much longer than that for the single-mode system. In a way the isolated two-mode system shows a relaxation behavior that is typical for open systems.

Turning on the dissipation leads to irreversible transfer to the lower state. However, the picture does not change qualitatively as the dissipative transfer mechanism does not contribute significantly at the early times shown here. As already found $\stackrel{46.47}{4}$ and seen in Fig. [ 7 the DDA has also no serious influence on the early dynamics. As the DDA has been recently well studied we have put the focus here on the ultrafast initial stage where the difference between the single-mode model and the two-mode model is best characterized.

\section{EFFICIENCY AND STABILITY}

To estimate the convergence and hence the stability of the proposed scheme one needs an appropriate measure for the convergence. Unraveling the QME one aims to calculate the time evolution of an observable, e.g. the population $\overline{P_{1}}$, performing an average over $N_{\mathrm{s}}$ single trajectories. At time $t_{j}$ the average reads

$$
\overline{P_{1}}\left(t_{j}, N_{\mathrm{s}}\right)=\frac{1}{N_{\mathrm{s}}} \sum_{i=1}^{N_{\mathrm{s}}}\left[\left\langle\psi_{i}\left(t_{j}\right) \mid 1\right\rangle\left\langle 1 \mid \phi_{i}\left(t_{j}\right)\right\rangle+\text { c.c. }\right] .
$$

As a convergence measure we introduce the quantity

$$
\varepsilon^{2}\left(N_{\mathrm{s}}, k\right)=\frac{1}{N_{t}} \sum_{j=1}^{N_{t}}\left[\overline{P_{1}}\left(t_{j}, N_{\mathrm{s}}\right)-\overline{P_{1}}\left(t_{j}, N_{\mathrm{s}}-k\right)\right]^{2},
$$

where $N_{t}$ is the total number of propagation time steps. In this convergence measure we use as reference the average performed over $N_{\mathrm{s}}-k$ trajectories. For convenience the increment $k$ can be chosen to be the number of computing nodes in a parallel implementation of the stochastic algorithm. One can easily see that $\varepsilon$ vanishes for large $N_{\mathrm{s}}$ if both terms in the sum converge to $\overline{P_{1}}\left(t_{j}, N_{\mathrm{s}} \rightarrow \infty\right)$. If these two terms diverge with increasing $N_{\mathrm{s}}$ the scheme is instable. Therefore $\varepsilon$ is sufficient for estimating the convergence and stability limits. The measure $\varepsilon$ used here is very similar to the absolute error measure $\beta$ for the unraveling schemes which has been used for studying standard first- and higher-order unraveling schemes ${ }^{8}$. The only difference between them is the reference calculation - for calculating the error $\beta$ one considers the exact solution produced numerically by some direct propagator or analytically. It was found $\frac{8}{\underline{8}}$ that for a small number of samples $N_{\mathrm{s}}$ the error measure $\beta$ is mainly statistical due to the finite sample size. For large $N_{\mathrm{s}}$ the error due to the finite time step starts to dominate. Since both terms in the sum in Eq. (41) carry the same time-step error it will cancel out in the error measure $\varepsilon$. Thus our convergence measure is a criterion for the statistical error of the stochastic scheme. 
Considering the efficiency of the stochastic method it is convenient to look at the relation between numerical effort and achieved convergence. When the DDA is invoked together with the RWA in the system-bath coupling one can obtain the Lindblad form (6) that can be solved by the standard quantum jump method 4.6.8.10.17 as well as by the present one. In this way the numerical performance of both methods can be compared. Figure 8 shows the behavior of $\varepsilon$ for $k=8$ with increasing number of trajectories for the ET example with one reaction coordinate. Within Redfield theory the variation of $\Gamma$ towards large system-bath coupling values is of limited validity. So the Redfield QME does not allow to verify the new scheme in the strong coupling regime. However, we performed the calculations for two values for $\Gamma\left(0.1 \omega_{0}\right.$ and $\left.0.01 \omega_{0}\right)$ that are supposed to belong to the weak coupling regime. In addition, the computation was performed for two propagation time steps $\delta t$. It was found that for $\Gamma \delta t \approx 0.1$ the proposed scheme becomes unstable. As can be seen in Fig. [ 8 decreasing $\Gamma \delta t$ has no influence on the convergence $\varepsilon$. The slope of $\varepsilon$ in the double logarithmic scale $\left(d \log \varepsilon / d \log N_{\mathrm{s}}\right)$ in Fig. 8 is -1 , i.e. the scheme converges as $1 / N_{\mathrm{s}}$. When simulating other, physically different systems we expect the convergence behavior to be not too different from the example studied here. At least the proportionality to $1 / N_{\mathrm{s}}$ will stay unchanged. The only difference can be the intercept of $\varepsilon$ (i.e. the value of $\log$ epsilon for $\log N_{\mathrm{s}}=0$ ) which may have some physical reasoning. We expect some change in the instability limit for $\Gamma \delta t \approx 0.1$ with the type of system studied. This topic has to be explored in future studies, especially for QMEs which allow larger variation of the system-bath coupling.

Now let us discuss the dependence on the basis size. It is well known that the numerical effort for solving QMEs with the quantum jump method and with direct propagators scales quadratically and cubically, respectively, with the basis size $\mathcal{N}$. Thus, for both high accuracy and lower numerical expense stochastic methods would only be preferred over direct propagators if the number of trajectories $N_{\mathrm{s}}$ is much smaller than $\mathcal{N}$. We shall examine the scaling behavior by solving the one-dimensional ET model with the use of the present stochastic scheme for both the Redfield QME (3) and the Lindblad QME (6) as well as the standard scheme for the Lindblad QME (6). All runs were performed with increasing basis size and compared to a direct propagation. For this purpose we choose the short iterative Arnoldi propagator ${ }^{11.22 .50}$ in a Krylov space of dimension 12. As expected, the numerical expense for a few trajectories is much smaller than for the direct propagator. But for converged results one needs much more that one trajectory. When the number of trajectories necessary for the complete convergence is greater than $\mathcal{N}$ one has to make a trade-off between the accuracy achieved with the stochastic method and the numerical effort. For the comparison we choose as an example $N_{\mathrm{s}}=500$ for which the convergence is not yet complete. In Fig. 2 we have seen that this sample size already yields qualitatively the same result as the direct propagator. For very accurate calculations one needs a much larger number of trajectories. The crossing point in Fig. 9 shows that for $\mathcal{N} \gtrsim 212$ the new stochastic algorithm should be preferred. In addition one gets a benefit from the efficient parallel implementation of the stochastic algorithm which is of great practical use especially when one is asking for fast preliminary results for the dynamics of large systems. The slope of the lines in Fig. 9 reflecting the scaling of the numerical effort for the stochastic methods is approximately 2.3 and for the direct propagation 3.2. These scalings are larger than the theoretical estimates of 2 and 
3 , respectively, because the true complexity of the algorithm is not a simple power function but a polynomial function of $\mathcal{N}$ due to array operations of lower order. This difference has to disappear for very large $\mathcal{N}$.

In the following the intercept of the curves for the numerical effort in Fig. 9, i.e. the CPUtime extrapolated to $\mathcal{N}=1$, is examined. The ratio of the intercepts between the proposed stochastic scheme for the Redfield QME and the standard scheme for the Lindblad QME is found to be 2.4 (see Fig. (9)). The respective ratio between the proposed stochastic scheme for the Redfield QME and the same scheme for the Lindblad QME is 4.4. This can be interpreted as follows. Neglecting the operations of lower order the intercept in a double logarithmic plot like in Fig. 9 has to be proportional to the number of matrix-vector multiplications for one time step, i.e. the intercept holds to some extent information specific for each stochastic scheme. Considering only the deterministic part of the implemented algorithm (see the Appendix for details) each time step requires $4 M+9$ matrix-vector multiplications (for $M$ dissipative channels, $4 \times M$ operations for computing the jump rates Eq. (201), eight operations for time propagation and one for calculating the population) versus only 5 for the standard jump algorithm (four operations for time propagation and one for calculating the population). Nevertheless, the values found from the simulation (Fig. 9), 12/5 and 22/5, deviate from the estimates $13 / 5$ and $17 / 5$, respectively, due to systematic effects like lowerorder operations for not very large $\mathcal{N}$ as well as due to stochastic effects like the varying number of quantum jumps. To summarize, for the Lindblad QME the standard algorithm is the method of choice, while for the Redfield QME, where one cannot apply the standard method, one has to cope with the larger numerical expense. The latter can be significantly reduced when high accuracy is not necessary.

\section{CONCLUSION}

In this paper the stochastic unraveling technique was extended for non-Lindblad QMEs. This progress became possible with the use of the wave-function pair in the doubled Hilbert space and the derivation of stable, almost normalized SSEs. An efficient first-order quantum jump algorithm was proposed. The efficiency is determined by the behavior of the norm of every single trajectory. In this sense the jump rates were used as parameters to influence the efficiency.

Occurrence of negative population for single trajectories is by no means a problem of the proposed unraveling scheme. Rather it is related to the fact that the QMEs (33) 7) do not preserve the RDM positive semi-definite. It is known $\underline{\underline{14}}$ that the negative eigenvalues of the $\mathrm{RDM}$ in the Redfield theory arise from the inconsistency between the initial RDM and the bath state, i.e. due to neglected initial correlations in the Born-Markov approximation. A satisfactory resolution of this problem is the slippage of the initial conditions as derived by Gaspard et al. $\underline{\underline{14}}$. In this method the so called slippage superoperator takes into account the short-time bath correlations. Applied to the initial RDM it introduces the necessary correlations into the initial state. This manipulation of the initial state ensures propagation of a positive semidefinite RDM at any further moment of time.

The method proposed was successfully tested for the Redfield QME for the damped 
harmonic oscillator and two ET models. It was shown that the scheme allows for more efficient quantum dynamical simulations of large systems. The most important benefit of the method is that it can be applied in a straightforward manner since the SSEs and the expressions of the jump rates are formally the same for other models of system and bath. Therefore, a potential use of the proposed method can be made in simulations involving any kind of non-Markovian QMEs provided that they are in a time-local form like in the time-convolutionless formalism ${ }^{25}$ as well as in methods using auxiliary density matrices to include the memory effects ${ }^{24}$.

Each individual trajectory occupies nearly the whole volume in phase space accessible for the system. It would be of possible interest to see whether the phase-space volume of a single trajectory shrinks with decreasing $\hbar$ and to study the classical behavior of the ET system.

\section{Acknowledgments}

The authors acknowledge financial support from the DFG.

\section{APPENDIX: THE NUMERICAL ALGORITHM}

This algorithm gives the numerical solution of the SSEs (15a) and (15b). The initial wave functions $\left|\psi_{i, s}(0)\right\rangle$ and $\left|\phi_{i, s}(0)\right\rangle$ are constructed so that

$$
\rho_{\mathrm{S}}(0)=\sum_{i=1}^{N_{e}} w_{i} \frac{1}{N_{\mathrm{S}}} \sum_{s=1}^{N_{\mathrm{s}}}\left[\left|\phi_{i, s}(0)\right\rangle\left\langle\psi_{i, s}(0)|+| \psi_{i, s}(0)\right\rangle\left\langle\phi_{i, s}(0)\right|\right] .
$$

Here $N_{e}$ is the number of non-zero eigenvalues $w_{i}$ of the initial density matrix $\rho_{\mathrm{s}}(0)$ and $N_{\mathrm{s}}$ the number of trajectories corresponding to each eigenvalue $w_{i}$. The wave functions are propagated jointly (as pairs) as follows starting with $t=0$.

1. store/send $\left|\psi_{i, s}(t)\right\rangle$ and $\left|\phi_{i, s}(t)\right\rangle$ for averaging;

2. calculate the rates $p_{k}^{1}$ and $p_{k}^{2}$ according to Eqs. (20a) and (20b).

3. generate a random number $\epsilon \in(0,1)$;

4. if $\epsilon>d t \sum_{k}\left(p_{k}^{1}+p_{k}^{2}\right)$ then

$$
\begin{aligned}
& \text { propagate }\left|\psi_{i, s}(t)\right\rangle \text { and }\left|\phi_{i, s}(t)\right\rangle \text { : } \\
& \quad \text { * find }\left|\psi_{i, s}(t+d t)\right\rangle \text { and }\left|\phi_{i, s}(t+d t)\right\rangle \text { solving } \\
& \quad d\left|\psi_{i, s}(t)\right\rangle / d t=A\left|\psi_{i, s}(t)\right\rangle \text { and } d\left|\phi_{i, s}(t)\right\rangle / d t=A\left|\phi_{i, s}(t)\right\rangle \text {, respectively } \\
& \quad * \text { set } t=t+d t \\
& \quad * \text { go to step } 1 \\
& \text { else } \\
& \quad * \text { if } \epsilon \leq d t \sum_{k} p_{k}^{1} \text { then }
\end{aligned}
$$


- jump with probability $p_{k}^{1} d t$ :

$$
\left|\psi_{i, s}(t)\right\rangle \rightarrow E_{k}\left|\psi_{i, s}(t)\right\rangle / \sqrt{p_{k}^{1}} \text { and }\left|\phi_{i, s}(t)\right\rangle \rightarrow C_{k}\left|\phi_{i, s}(t)\right\rangle / \sqrt{p_{k}^{1}}
$$

else

- jump with probability $p_{k}^{2} d t$ :

$$
\left|\psi_{i, s}(t)\right\rangle \rightarrow C_{k}\left|\psi_{i, s}(t)\right\rangle / \sqrt{p_{k}^{2}} \text { and }\left|\phi_{i, s}(t)\right\rangle \rightarrow E_{k}\left|\phi_{i, s}(t)\right\rangle / \sqrt{p_{k}^{2}}
$$

* go to step 2

The deterministic propagation of the wave functions in this work was performed with the use of a forth-order Runge-Kutta method $\underline{62}$. Accordingly, one time step requires four matrixvector multiplication for each wave function. The ensemble-averaged expectation value of an observable $A$ is calculated as

$$
\overline{\langle A(t)\rangle}=\sum_{i=1}^{N_{e}} w_{i} \frac{1}{N_{\mathrm{s}}} \sum_{s=1}^{N_{\mathrm{s}}}\left[\left\langle\psi_{i, s}(t)|A| \phi_{i, s}(t)\right\rangle+\text { c.c. }\right] .
$$

The method can be parallelized using $\mathrm{MPI}^{63}$. In such an implementation every single stochastic trajectory is propagated by a different process. Only the averaging operation (43) is done at certain times by means of collective communications. In this way the task can be efficiently distributed on a cluster of PCs.

* Present address: Theoretische Chemie, Technische Universität München, 85747 Garching, Germany

1 U. Weiss, Quantum Dissipative Systems (World Scientific, Singapore, 1999), 2nd ed.

2 V. May and O. Kühn, Charge and Energy Transfer Dynamics in Molecular Systems (WileyVCH, Berlin, 2000).

3 F. E. van Dorsselaer and G. Nienhuis, J. Opt. B: Quantum Semiclass. Opt. 2, L5 (2000).

4 J. Dalibard, Y. Castin, and K. Mølmer, Phys. Rev. Lett. 68, 580 (1992).

5 K. Mølmer, Y. Castin, and J. Dalibard, J. Opt. Soc. Am. B 10, 524 (1993).

6 C. W. Gardiner, A. S. Parkins, and P. Zoller, Phys. Rev. A 46, 4363 (1992).

7 H. Carmichael, An Open System Approach to Quantum Optic (Springer, Berlin, 1993).

8 J. Steinbach, B. M. Garraway, and P. L. Knight, Phys. Rev A 51, 3302 (1995).

9 N. Gisin and I. C. Percival, J. Phys. A: Math. Gen. 25, 5677 (1992).

10 M. B. Plenio and P. L. Knight, Rev. Mod. Phys. 70, 101 (1998).

11 I. Kondov, U. Kleinekathöfer, and M. Schreiber, J. Chem. Phys. 114, 1497 (2001).

12 G. Lindblad, Commun. Math. Phys. 40, 147 (1975).

13 P. Pechukas, Phys. Rev. Lett. 73, 1060 (1994).

14 P. Gaspard and M. Nagaoka, J. Chem. Phys. 111, 5668 (1999).

15 S. L. Adler, Phys. Lett. A 265, 58 (2000).

16 J. Wilkie, Phys. Rev. E 62, 8808 (2000).

17 B. M. Garraway and P. L. Knight, Phys. Rev. A 49, 1266 (1994). 
18 B. Wolfseder and W. Domcke, Chem. Phys. Lett. 235, 370 (1995).

19 W. T. Strunz, L. Diósi, N. Gisin, and T. Yu, Phys. Rev. Lett. 83, 4909 (1999).

20 F. Haake and R. Reibold, Phys. Rev. A 32, 2462 (1985).

21 W. T. Strunz, Chem. Phys. 268, 237 (2001).

22 W. T. Pollard, A. K. Felts, and R. A. Friesner, Adv. Chem. Phys. 93, 77 (1996).

23 V. Ćápek, Physica A 203, 520 (1994).

24 C. Meier and D. J. Tannor, J. Chem. Phys. 111, 3365 (1999).

25 H.-P. Breuer, B. Kappler, and F. Petruccione, Phys. Rev. A 59, 1633 (1999).

26 P. Gaspard and M. Nagaoka, J. Chem. Phys. 111, 5676 (1999).

27 J. T. Stockburger and H. Grabert, Phys. Rev. Lett. 88, 170407 (2002).

28 H.-P. Breuer, B. Kappler, and F. Petruccione, Phys. Rev. A 56, 2334 (1997).

29 U. Kleinekathöfer, I. Kondov, and M. Schreiber, Phys. Rev. E 66, 037701 (2002).

30 I. Goychuk, L. Hartmann, and P. Hänggi, Chem. Phys. 268, 151 (2001).

31 N. Makri, E. Sim, D. Makarov, and M. Topaler, Proc. Natl. Acad. Sci. USA 93, 3926 (1996).

32 E. Sim and N. Makri, J. Phys. Chem. B 101, 5446 (1997).

33 M. Thoss, H. Wang, and W. H. Miller, J. Chem. Phys. 115, 2991 (2001).

34 M. Thoss and H. Wang, Chem. Phys. Lett. 358, 298 (2002).

35 H. Wang and M. Thoss, Isr. J. Chem. (2003), (in press).

36 V. May and M. Schreiber, Phys. Rev. A 45, 2868 (1992).

37 J. M. Jean, R. A. Friesner, and G. R. Fleming, J. Chem. Phys. 96, 5827 (1992).

38 A. K. Felts, W. T. Pollard, and R. A. Friesner, J. Phys. Chem. 99, 2929 (1995).

39 C. Fuchs and M. Schreiber, J. Chem. Phys. 105, 1023 (1996).

40 B. Wolfseder and W. Domcke, Chem. Phys. Lett. 259, 113 (1996).

41 P. F. Barbara, T. J. Meyer, and M. A. Ratner, J. Phys. Chem. 100, 13148 (1996).

42 M. Bixon and J. Jortner, Adv. Chem. Phys. 106\&107, 35 (1999).

43 V. Čápek, Physica A 203, 495 (1994).

44 V. Ćápek and J. Peřina, Jr, Physica A 215, 209 (1995).

45 T. Mančal and V. May, Chem. Phys. 268, 201 (2001).

46 D. Egorova, A. Kühl, and W. Domcke, Chem. Phys. 268, 105 (2001).

47 U. Kleinekathöfer, I. Kondov, and M. Schreiber, Chem. Phys. 268, 121 (2001).

48 M. Schreiber, I. Kondov, and U. Kleinekathöfer, J. Lumin. 94\&95, 471 (2001).

49 D. Egorova, M. Thoss, W. Domcke, and H. Wang, J. Chem. Phys. (2003), (in press).

50 W. T. Pollard and R. A. Friesner, J. Chem. Phys. 100, 5054 (1994).

51 C. W. Gardiner, Handbook of Stochastic Methods (Springer, Berlin, 1985).

52 T. Felbinger and M. Wilkens, J. Mod. Opt. 46, 1401 (1999).

53 B. Wolfseder, Dissertation, Technische Universität München, Theoretische Chemie, TU München, 85747 Garching (1997).

54 D. Kohen, C. C. Marston, and D. J. Tannor, J. Chem. Phys. 107, 5236 (1997).

55 O. Linden and V. May, Eur. Phys. J. D 12, 473 (2000).

56 R. Dum, A. S. Parkins, P. Zoller, and C. W. Gardiner, Phys. Rev. A 46, 4382 (1992).

57 M. Hillery, R. F. 0'Connell, M. O. Scully, and E. P. Wigner, Phys. Rep. 106, 121 (1984).

58 B. Wolfseder, L. Seidner, W. Domcke, G. Stock, M. Seel, S. Engleitner, and W. Zinth, Chem. 
Phys. 233, 323 (1998).

59 S. A. Kovalenko, N. Eilers-König, T. A. Senyushkina, and N. P. Ernsting, J. Phys. Chem. A 105, 4834 (2001).

60 M. C. M. O'Brien, J. Phys. C 5, 2045 (1972).

61 T. Takagahara, E. Hanamura, and R. Kubo, J. Phys. Soc. Japan 44, 728 (1978).

62 W. H. Press, S. A. Teukolsky, W. T. Vetterling, and B. P. Flannery, Numerical Recipes in FORTRAN 77 (Cambridge University Press, Cambridge, 1992).

63 M. P. I. Forum, Tech. Rep. CS-94-230, Computer Science Dept., University of Tennessee, Knoxville, TN (1994), available online at http://www-unix.mcs.anl.gov/mpi/index.html. 


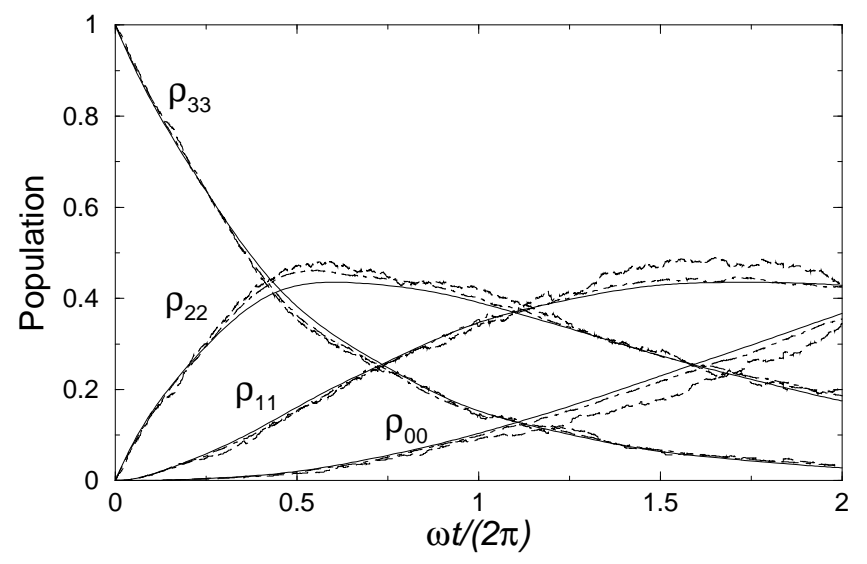

FIG. 1: Population dynamics of a damped harmonic oscillator simulated using the proposed quantum jump method with $10^{3}$ trajectories (dashed lines) and with $10^{4}$ trajectories (dot-dashed lines) compared to the direct RDM propagation (solid lines).

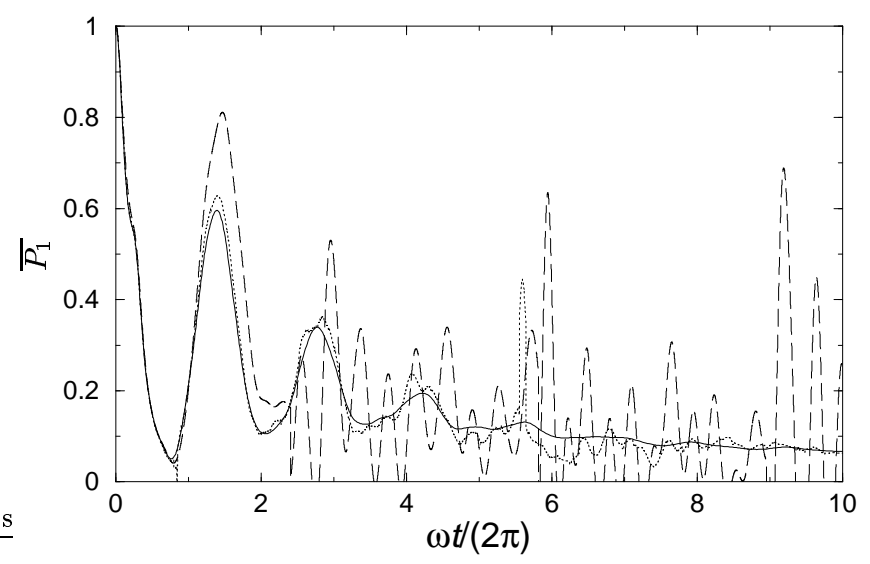

FIG. 2: Relaxation of the donor population for the electron transfer model with a single reaction mode. The solid line shows the exact solution of the QME, the dashed line one arbitrary trajectory of the quantum jump method, the dotted line an average over 500 trajectories. 


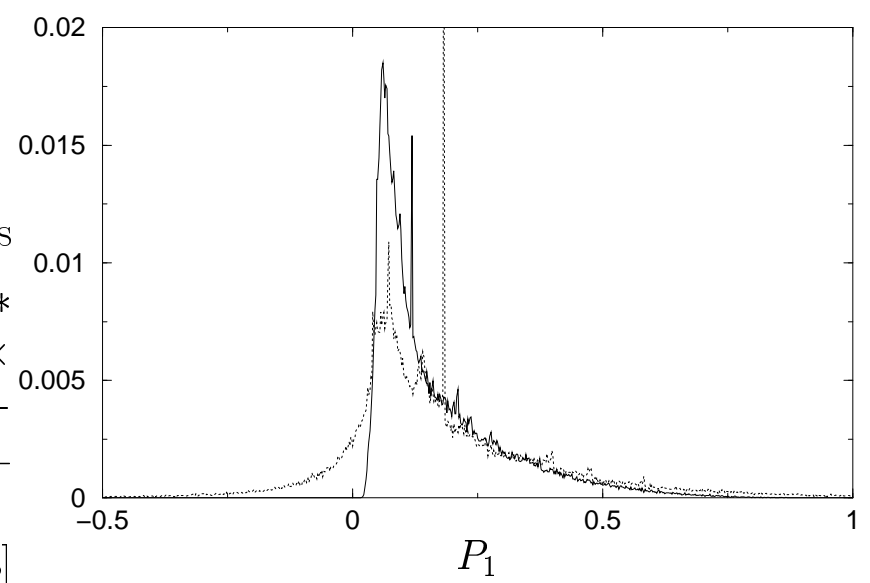

FIG. 3: Distribution of the expectation values of the population of the donor state $P_{1}$ produced by the new unraveling scheme for the Redfield QME (dotted line) and the standard normalized jump method for the Lindblad QME (solid line) at time $\omega t /(2 \pi)=5.8$. Both distributions are normalized to unity. 

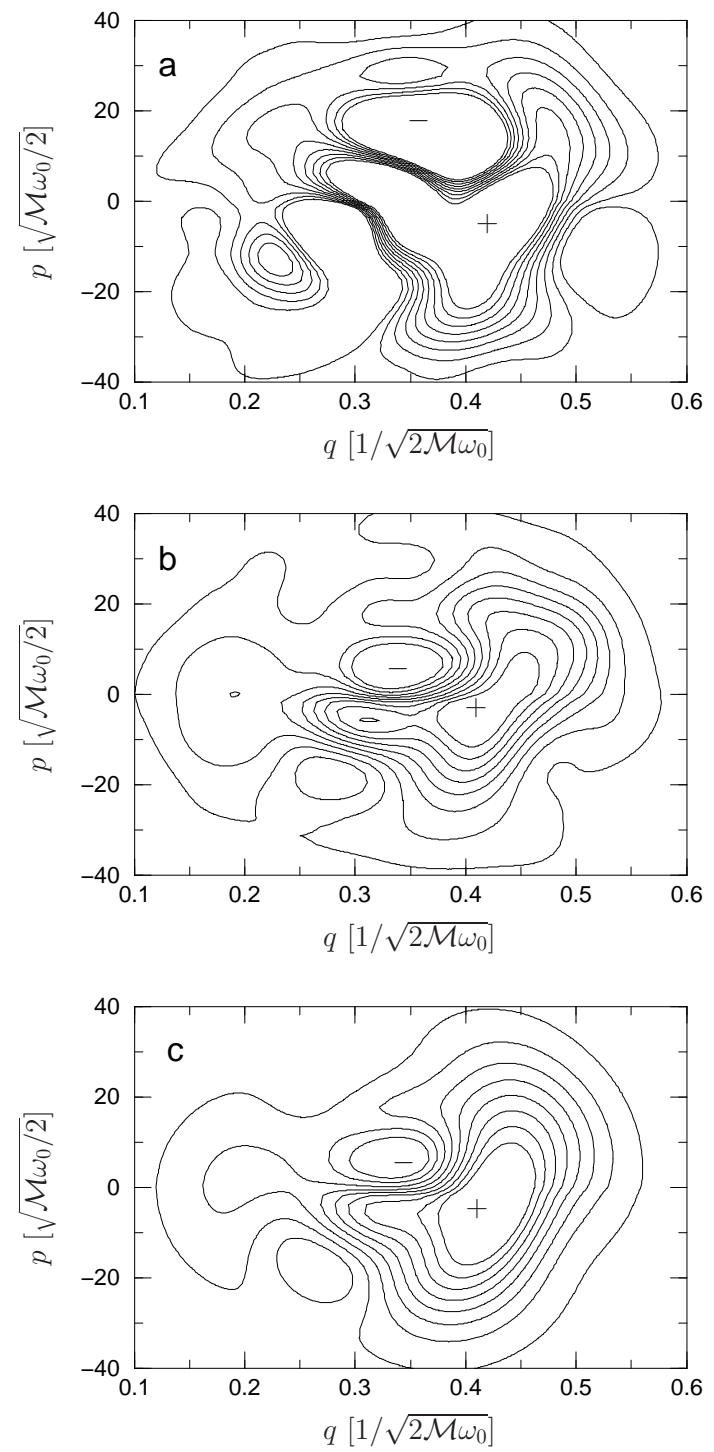

FIG. 4: Contour plots of the Wigner representation of the RDM of one individual trajectory (a), of the RDM recovered with 500 trajectories (b) and of the exact RDM (c). Regions with maximum are denoted with + , and regions with minimum with - . 


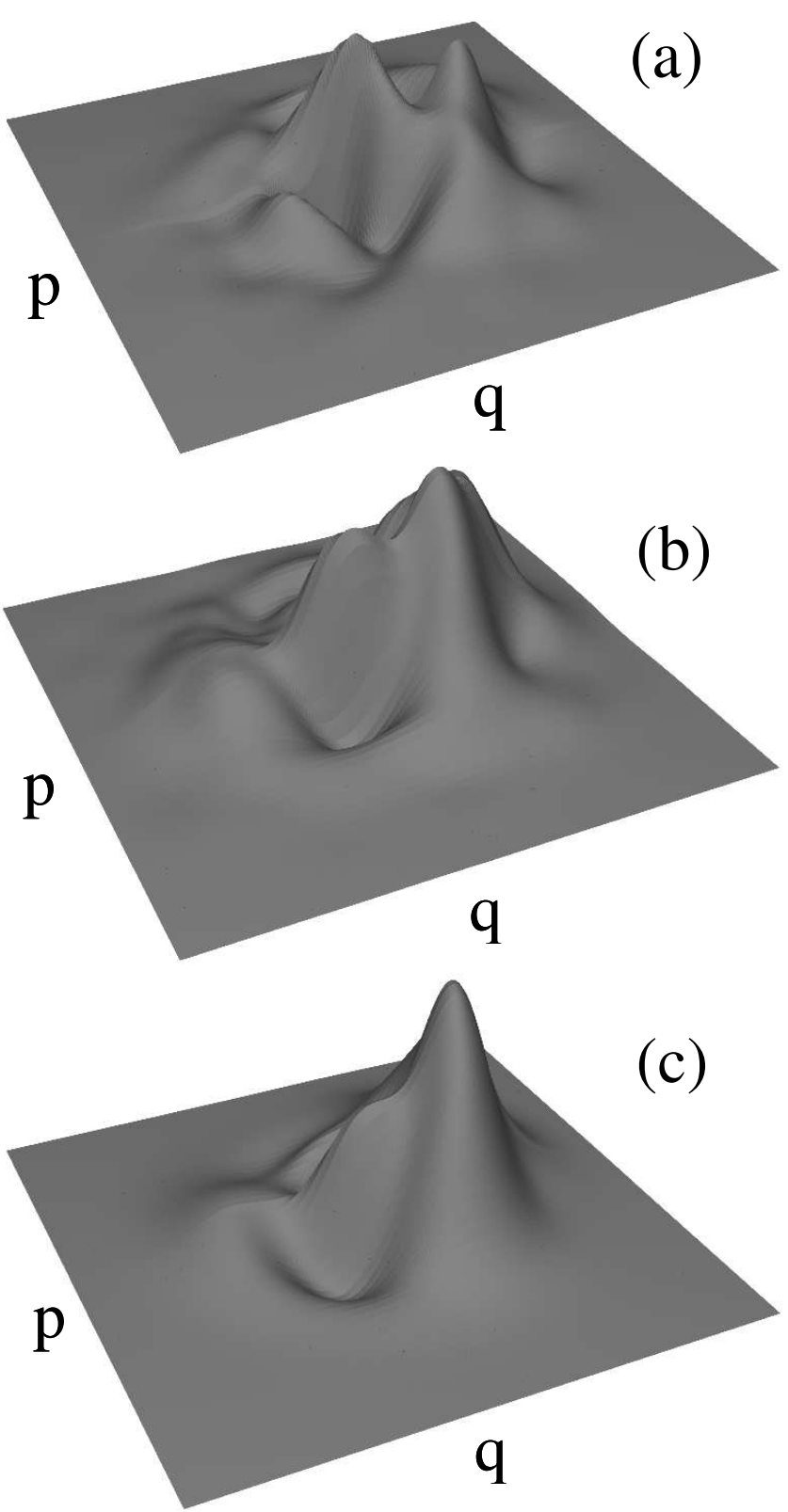

FIG. 5: Three-dimensional plots of the Wigner representation of the RDM of one individual trajectory (a), of the RDM recovered with 500 trajectories (b) and of the exact RDM (c). The data shown here are the same as in Fig. 4 


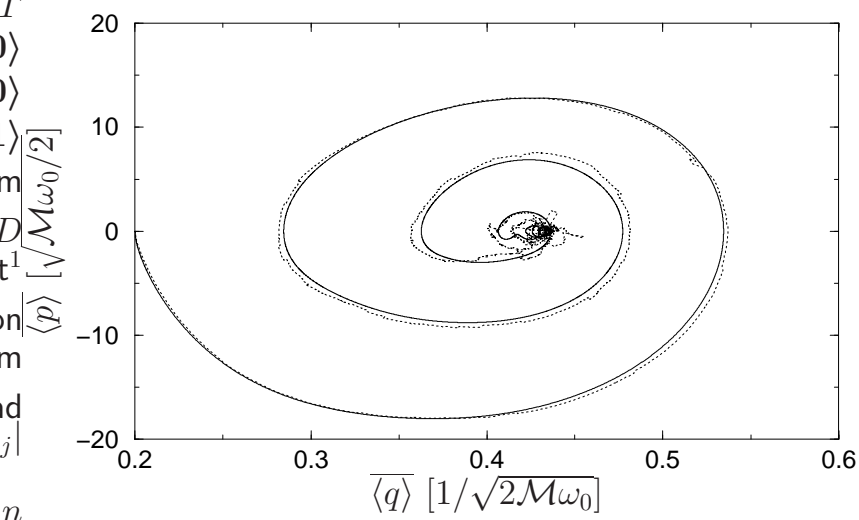

FIG. 6: Dynamics of ET depicted as a path in phase space calculated by the exact solution of the Redfield QME (solid line) and by averaging over 500 trajectories of the quantum jump method (dotted line).

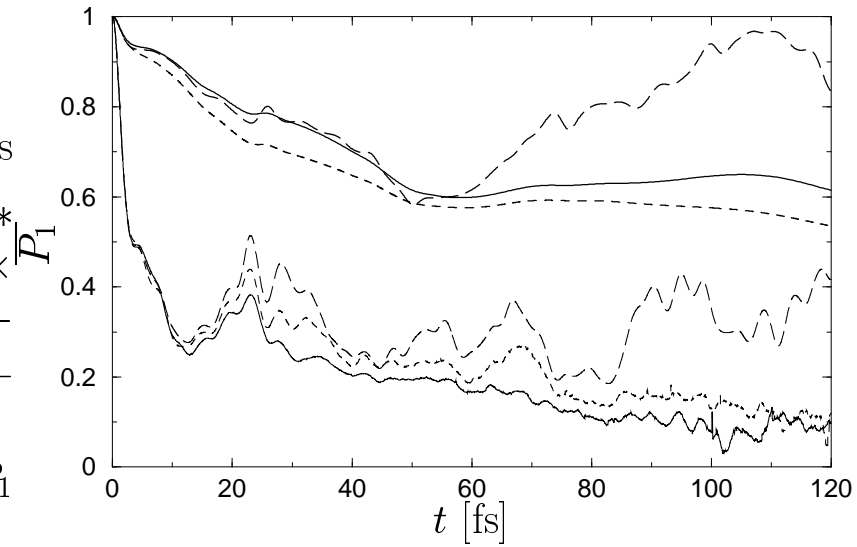

FIG. 7: Population dynamics of the excited state in the effective one-mode (thick lines) and the two-mode (thin lines) models for ET. Coherent dynamics are denoted by long dashed lines, dynamics with dissipation in DDA by dashed lines, and the Redfield dynamics by solid lines. The two-mode model is solved with the new stochastic method with 5000 trajectories. 


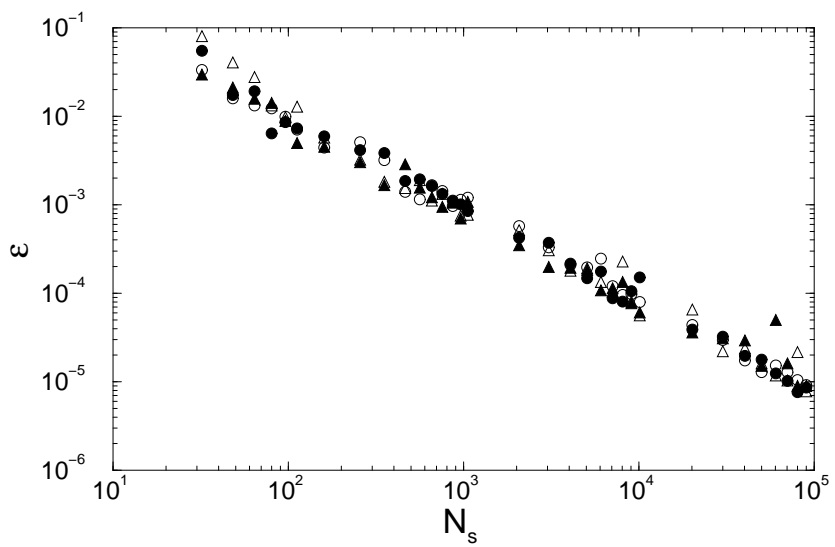

FIG. 8: Convergence behavior of the proposed stochastic unraveling scheme for $\Gamma=0.01 \omega_{0}$ (opaque), $\Gamma=0.1 \omega_{0}$ (filled), $\delta t=1$ (circles), and $\delta t=10$ (triangles).

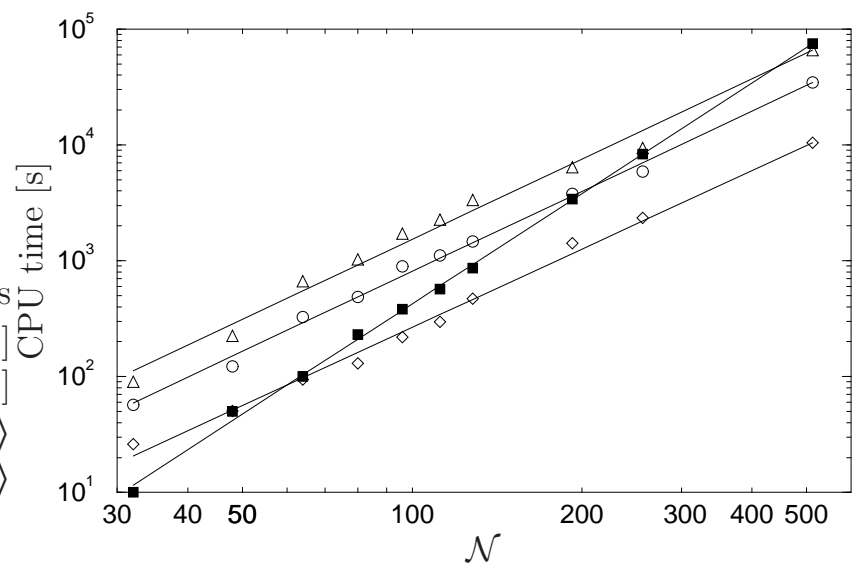

FIG. 9: Numerical effort of the new unraveling scheme with 500 trajectories for Eq. (3) (circles), the standard quantum jump method for Eq. (6) (rhombs), and the new scheme for Eq. (6) (triangles) shown for the model of one-dimensional ET. The short iterative Arnoldi method (filled squares) is shown as reference solving Eq. (3). The data points at $\mathcal{N}=512$ are extrapolated. 